\title{
ON THE CONVERGENCE ANALYSIS OF THE SPLINE COLLOCATION METHOD FOR SYSTEM OF INTEGRAL ALGEBRAIC EQUATIONS OF INDEX-2
}

\author{
F. GHOREISHI, M. HADIZADEH* and S. PISHBIN \\ Department of Mathematics \\ K. N. Toosi University of Technology \\ Tehran, Iran \\ *hadizadeh@kntu.ac.ir
}

\author{
Received 24 October 2011 \\ Accepted 29 May 2012 \\ Published 14 December 2012
}

\begin{abstract}
This article presents some theoretical results for polynomial spline collocation solution to a new class of semi-explicit Integral Algebraic Equations (IAEs) of index-2, which has been introduced in a recent paper of the authors (Hadizadeh, M., Ghoreishi, F. and Pishbin, S. [2011] "Jacobi spectral solution for integral-algebraic equations of index-2," Appl. Numer. Math. 61, 131-148). Critical issues for numerical analysis of the spline collocation method for this type of Volterra systems are discussed and the necessary and sufficient conditions are presented which guarantee the convergence of the method. We analyze the rate of convergence for two disjoint cases of collocation parameter $c_{m}$. Numerical results confirm the rate of decay of the error predicted by this theory.
\end{abstract}

Keywords: Integral algebraic equation; system of Volterra integral equation; index of IAEs; spline collocation method; error analysis; numerical treatment.

AMS Subject Classification: 65R20, 45F15.

\section{Introduction}

The principal aim of this paper is to serve the numerical analysis of the spline collocation method for a mixed system of Volterra integral equations of the first and second kind which is known as Integral Algebraic Equations (IAEs). In fact, the present work is concerned with the semi-explicit system of integral algebraic equations of index-2

$$
\left\{\begin{array}{l}
y(t)=f(t)+\left(\nu_{11} y\right)(t)+\left(\nu_{12} z\right)(t), \\
0=g(t)+\left(\nu_{21} y\right)(t),
\end{array}\right.
$$

*Corresponding author. 
where the Volterra integral operators $\nu_{k l}$ are given by

$$
\left(\nu_{k l} \varphi\right)(t)=\int_{0}^{t} K_{k l}(t, s) \varphi(s) d s, \quad t \in I=[0, T], \quad(k, l=1,2),
$$

and $y, f: I \rightarrow \mathbb{R}^{d_{1}}, z, g: I \rightarrow \mathbb{R}^{d_{2}}, K_{k k}(.,.) \in L\left(\mathbb{R}^{d_{k}}\right), K_{12}(.,.) \in L\left(\mathbb{R}^{d_{2}}\right.$, $\left.\mathbb{R}^{d_{1}}\right), K_{21}(.,.) \in L\left(\mathbb{R}^{d_{1}}, \mathbb{R}^{d_{2}}\right)$ are continuous functions and $L(.,$.$) is the linear trans-$ formation space. The system (1) is a special case of the following general form of IAEs

$$
A(t) X(t)=G(t)+\int_{0}^{t} K(t, s, X(s)) d s, \quad t \in I=[0, T],
$$

where $A(t)$ is a singular matrix with continuous entries $(\operatorname{rank}(\mathrm{A}) \geq 1, \operatorname{det}(A)=0)$ and $G(t)$ is a known free term.

Such equations and systems frequently arise in many physical and applied problems especially in the fields of viscoelastic materials [Janno and Wolfersdorf (1997) and Zenchuk (2008)], dynamic processes in chemical reactors [Kafarov et al. (1999)], identification of memory kernels in heat conduction [Wolfersdorf (1994)], evolution of a chemical reaction within a small cell [Jumarhon et al. (1996)] and the two-dimensional biharmonic equation in a semi-infinite strip [Cannon (1984) and Gomilko (2003)]. A primary and general theory of IAEs are due to Chistyakov and Gear in 1986-1990, who determined the theory and difficulties of these equations [see, for instance Chistyakov (1986); Gear (1990)]. A system of IAEs is assigned by a number which is known as index, to measure its complexity concerning both theoretical and numerical treatments. Actually, the index plays a key role in the solvability and regularity of the solution of IAEs.

There has been few work on analyzing and numerical methods for the IAEs in literature. The existence and uniqueness results of solution for IAEs systems with convolutions kernels have been discussed in Bulatov and Chistyakov [1997]. Kauthen [2000] applied the polynomial spline collocation method for a semi-explicit IAEs with index-1 and established global convergence as well as local superconvergence. Brunner [2004] defined the index-1 tractable for a semi-explicit form of IAEs and investigated the existence of a unique solution for this type of systems. Recently, the authors in Hadizadeh et al. [2011] have defined the index-2 tractable for a new class of IAEs and proposed a Jacobi collocation method including the matrix-vector multiplication representation of the equation. Most recently, the numerical analysis of the two-dimensional integral-algebraic system has also investigated by Bulatov and Lima in [2011].

As we mentioned, the solution of IAEs has been closely related to the definition of index. Different definitions of index have been given in the literature [see, for instance Gear (1990); Brunner (2004); Hadizadeh et al. (2011)]. The "index reduction procedure" for IAEs systems has been introduced by Gear [1990], in which if the process is terminated, then the index is determined. This means that under suitable conditions, there is a solution for the resulting regular system of integral 
equations. Here, our mean about the index for the IAEs system (1), is the minimum number of differentiation of the second equation of (1) with respect to $t$, in order a system of regular VIEs has been reached. Generally, this methodology has several restrictions that can causes instability from a numerical point of view. Since the smoothness condition of the second equation of (1) is not often satisfied in some applications, we may consider the index notion without use of derivative arrays (e.g., the tractability index which is due to Brunner [2004] and the authors in Hadizadeh et al. [2011]). Also, it is worth mentioning that the numerical schemes which are applicable (i.e., convergent) for IAEs of a given index, might not be useful for IAEs of higher index. Note that the IAEs with index $>1$ are generally hard to solve and are still under active research.

Throughout this paper, we consider the case when the conditions of Theorem 1 in Hadizadeh et al. [2011] be satisfied. Hence, in virtue of the smoothness of the given functions, the existence, uniqueness and the regularity results for continuous solutions of the IAEs system (1) may be established from the following theorem:

Theorem 1 [From Hadizadeh et al. [2011]]. Let $\nu \geq 0$ and assume that

(1) $K_{1 l} \in C^{\nu}(D)$ for $l=1,2$ and $D=\{(t, s): 0 \leq s \leq t \leq T\}$,

(2) $K_{21} \in C^{\nu+1}(D)$ and $\left|\operatorname{det}\left(K_{21}(t, t) K_{12}(t, t)\right)\right| \geq k_{0}>0$,

(3) $f \in C^{\nu}(D), g \in C^{\nu+1}(D)$ and $g_{1}(0)=0$,

then the IAEs (1) possesses a unique solution $y, z \in C^{\nu}(I)$.

The main aim of this work is to provide the necessary and sufficient conditions for convergence of the spline collocation method for the IAEs system (1). For this purpose, we first apply the polynomial spline collocation method for the numerical solvability of the index-2 system of IAEs (1) in Sec. 2. A critical global convergence analysis of the method for two disjoint cases of collocation parameter $c_{m}$ is then given in Sec. 3 and finally in Sec. 4, some numerical experiments are reported which supporting the theoretical results.

\section{The Spline Collocation Method}

Let $\Pi_{N}=\left\{0=t_{0}<t_{1}<\cdots<t_{n}=T\right\}$ be a uniform partition of the interval $I=[0, T]$ with grid points $t_{n}=n h,(n=0, \ldots, N)$. Also, let $h$ be the stepsize and $\Theta$ is given by:

$$
\Theta=\left\{t_{n j}=t_{n}+c_{j} h: 0<c_{1}<c_{2}<\cdots<c_{m} \leq 1,0 \leq n \leq N-1\right\},
$$

where $c_{j}(j=1, \ldots, m)$ and $t_{n j}$ are the collocation parameters and the collocation points, respectively. We define the subintervals $\sigma_{n}=\left(t_{n}, t_{n+1}\right], n=0, \ldots, N-1$, and the space of piecewise polynomials of degree $m-1 \geq 0$, as follows:

$$
S_{m-1}^{-1}\left(\Pi_{N}\right)=\left\{v:\left.v\right|_{\sigma_{n}} \in \Pi_{m-1},(0 \leq n \leq N-1)\right\} .
$$

Consider the IAEs system (1) and approximate the solution $\left(y_{1}, \ldots, y_{d_{1}}\right.$, $\left.z_{1}, \ldots, z_{d_{2}}\right)^{T}$ by $(\mathbf{u}, \mathbf{v})^{T}$, where $\mathbf{u}=\left\{u_{l}\right\}_{l=1}^{d_{1}}, \mathbf{v}=\left\{v_{l}\right\}_{l=1}^{d_{2}}$ and each component 
of $\mathbf{u}$ and $\mathbf{v}$ belongs to $S_{m-1}^{-1}\left(\Pi_{N}\right)$. The collocation solution $\mathbf{u}, \mathbf{v}$ can be defined by the following collocation equations

$$
\begin{aligned}
\mathbf{u}(t) & =\mathbf{f}(t)+\int_{0}^{t} \mathbf{K}_{11}(t, s) \mathbf{u}(s) d s+\int_{0}^{t} \mathbf{K}_{12}(t, s) \mathbf{v}(s) d s \\
\mathbf{0} & =\mathbf{g}(t)+\int_{0}^{t} \mathbf{K}_{21}(t, s) \mathbf{u}(s) d s
\end{aligned}
$$

where $\mathbf{f}(t)=\left\{f_{l}(t)\right\}_{l=1}^{d_{1}}, \mathbf{g}(t)=\left\{g_{l}(t)\right\}_{l=1}^{d_{2}}, \mathbf{K}_{p q}(t, s)=\left\{K_{p q}(t, s)\right\}_{d_{p} \times d_{q}}, \quad(p, q=$ $1,2), \mathbf{0}=\{0\}_{d_{2} \times 1}$ and $t \in \Theta$.

Since $\left\{u_{l}\right\}_{l=1}^{d_{1}},\left\{v_{l}\right\}_{l=1}^{d_{2}} \in S_{m-1}^{-1}\left(\Pi_{N}\right)$, (for $\left.\rho \in(0,1]\right)$, the following relations hold:

$$
\begin{aligned}
& \mathbf{u}\left(t_{n}+\rho h\right)=\sum_{j=1}^{m} \mathbf{U}_{n j} L_{j}(\rho), \quad \mathbf{U}_{n j}=\mathbf{u}\left(t_{n}+c_{j} h\right), \\
& \mathbf{v}\left(t_{n}+\rho h\right)=\sum_{j=1}^{m} \mathbf{V}_{n j} L_{j}(\rho), \quad \mathbf{V}_{n j}=\mathbf{v}\left(t_{n}+c_{j} h\right),
\end{aligned}
$$

where $\mathbf{U}_{n j}=\left\{U_{l n j}\right\}_{l=1}^{d_{1}}, \mathbf{V}_{n j}=\left\{V_{l n j}\right\}_{l=1}^{d_{2}}$ and $L_{j}(\rho)$ represents the Lagrange canonical polynomials for the collocation parameters $\left\{c_{j}\right\}$ which is defined as:

$$
L_{j}(\rho)=\prod_{k \neq j} \frac{\left(\rho-c_{k}\right)}{\left(c_{j}-c_{k}\right)}, \quad j=1, \ldots, m .
$$

Let us set $\rho=\left(s-t_{i}\right) / h,(i=0, \ldots, n)$, and insert (4) and (5) into relations (2) and $(3)$, respectively, then we have

$$
\begin{aligned}
\mathbf{U}_{n j}= & \mathbf{f}\left(t_{n j}\right)+h \sum_{i=0}^{n-1} \sum_{k=1}^{m}\left(\int_{0}^{1} \mathbf{K}_{11}\left(t_{n j}, t_{i}+\rho h\right) \mathbf{U}_{i j} L_{k}(\rho) d \rho\right) \\
& +h \sum_{i=0}^{n-1} \sum_{k=1}^{m}\left(\int_{0}^{1} \mathbf{K}_{12}\left(t_{n j}, t_{i}+\rho h\right) \mathbf{V}_{i j} L_{k}(\rho) d \rho\right) \\
& +h \sum_{k=1}^{m}\left(\int_{0}^{c_{j}} \mathbf{K}_{11}\left(t_{n j}, t_{n}+\rho h\right) \mathbf{U}_{n j} L_{k}(\rho) d \rho\right) \\
& +h \sum_{k=1}^{m}\left(\int_{0}^{c_{j}} \mathbf{K}_{12}\left(t_{n j}, t_{n}+\rho h\right) \mathbf{V}_{n j} L_{k}(\rho) d \rho\right), \\
\mathbf{0}= & \mathbf{g}\left(t_{n j}\right)+h \sum_{i=0}^{n-1} \sum_{k=1}^{m}\left(\int_{0}^{1} \mathbf{K}_{21}\left(t_{n j}, t_{i}+\rho h\right) \mathbf{U}_{i j} L_{k}(\rho) d \rho\right) \\
& +h \sum_{k=1}^{m}\left(\int_{0}^{c_{j}} \mathbf{K}_{21}\left(t_{n j}, t_{n}+\rho h\right) \mathbf{U}_{n j} L_{k}(\rho) d \rho\right) .
\end{aligned}
$$


Note that, the integrals in (6) and (7) cannot be computed explicitly in general, and thus one has to use appropriate quadrature rules to approximate them.

$$
\begin{aligned}
\int_{0}^{c_{j}} \mathbf{K}_{p q}\left(t_{n j}, t_{n}+\rho h\right) L_{i}(\rho) d \rho & \approx \sum_{k=1}^{m} \mathbf{K}_{p q}\left(t_{n j}, t_{n}+c_{k} h\right) a_{j k}, \\
\int_{0}^{1} \mathbf{K}_{p q}\left(t_{n j}, t_{n}+\rho h\right) L_{i}(\rho) d \rho & \approx \sum_{k=1}^{m} \mathbf{K}_{p q}\left(t_{n j}, t_{n}+c_{k} h\right) b_{k},
\end{aligned}
$$

where $p, q=1,2$, and the coefficients $a_{j k}$ and $b_{k}$ are defined by

$$
a_{j k}=\int_{0}^{c_{j}} L_{k}(\rho) d \rho, \quad b_{k}=\int_{0}^{1} L_{k}(\rho) d \rho, \quad j, k=1, \ldots, m .
$$

By inserting the above relations into Eqs. (6) and (7), we get

$$
\begin{aligned}
\mathbf{U}_{n j}= & \mathbf{f}\left(t_{n j}\right)+h \sum_{i=0}^{n-1} \sum_{k=1}^{m} \mathbf{K}_{11}\left(t_{n j}, t_{i k}\right) \mathbf{U}_{i k} b_{k}+h \sum_{i=0}^{n-1} \sum_{k=1}^{m} \mathbf{K}_{12}\left(t_{n j}, t_{i k}\right) \mathbf{V}_{i k} b_{k} \\
& +h \sum_{k=1}^{m} \mathbf{K}_{11}\left(t_{n j}, t_{n k}\right) \mathbf{U}_{n k} a_{j k}+h \sum_{k=1}^{m} \mathbf{K}_{12}\left(t_{n j}, t_{n k}\right) \mathbf{V}_{n k} a_{j k}, \\
\mathbf{0}= & \mathbf{g}\left(t_{n j}\right)+h \sum_{i=0}^{n-1} \sum_{k=1}^{m} \mathbf{K}_{21}\left(t_{n j}, t_{i k}\right) \mathbf{U}_{i k} b_{k}+h \sum_{k=1}^{m} \mathbf{K}_{21}\left(t_{n j}, t_{n k}\right) \mathbf{U}_{n k} a_{j k} .
\end{aligned}
$$

Now, by substituting $\mathbf{U}_{n j}$ and $\mathbf{V}_{n j}$ as solution of the resulting system into (4) and (5), we can get the numerical solution of IAEs system (1) for arbitrary $\rho \in(0,1]$.

\section{Convergence Analysis}

In this section, we give the main result of this paper in order to preserve the global convergence of the proposed scheme. The analysis employed here is essentially relying on the given approach of the polynomial spline collocation method for the system of IAEs of index-1 in Kauthen [2000] including some innovations and new initiatives in the case $c_{m}<1$, which lead to completely different order of convergence results.

Theorem 2. Assume that $\mathbf{u}=\left(u_{1}, \ldots, u_{d_{1}}\right)^{T}$ and $\mathbf{v}=\left(v_{1}, \ldots, v_{d_{2}}\right)^{T}$ with $\left\{u_{l}\right\}_{l=1}^{d_{1}},\left\{v_{l}\right\}_{l=1}^{d_{2}} \in S_{m-1}^{-1}\left(\Pi_{N}\right)$ are the collocation approximations to the solution $\mathbf{y}=\left(y_{1}, y_{2}, \ldots, y_{d_{1}}\right)^{T}, \mathbf{z}=\left(z_{1}, z_{2}, \ldots, z_{d_{2}}\right)^{T}$ of the index-2 IAEs system (1) which is defined by (4) and (5) and let the hypotheses of Theorem 1 hold. For the collocation parameter $c_{m}$, two following cases can be considered:

(1) If $c_{m}=1$, the collocation approximation $(\mathbf{u}, \mathbf{v})^{T}$ converges to the solution $(\mathbf{y}, \mathbf{z})^{T}$, and we have

$$
\|\mathbf{y}-\mathbf{u}\|_{\infty}=O\left(h^{m}\right), \quad\|\mathbf{z}-\mathbf{v}\|_{\infty}=O\left(h^{m-1}\right) .
$$


(2) If $c_{m}<1$, the collocation approximation $(\mathbf{u}, \mathbf{v})^{T}$ converges to the solution $(\mathbf{y}, \mathbf{z})^{T}$ for any $m \geq 3$, if and only if

$$
-1 \leq \rho_{m}=(-1)^{m} \prod_{i=1}^{m} \frac{1-c_{i}}{c_{i}} \leq 1
$$

Furthermore, the following order of convergence holds:

$$
\begin{aligned}
& \|\mathbf{y}-\mathbf{u}\|_{\infty}= \begin{cases}O\left(h^{m}\right), & \text { if } \rho_{m} \in[-1,1), \\
O\left(h^{m-1}\right), & \text { if } \rho_{m}=1,\end{cases} \\
& \|\mathbf{z}-\mathbf{v}\|_{\infty}= \begin{cases}O\left(h^{m-1}\right), & \text { if } \rho_{m} \in(-1,1), \\
O\left(h^{m-2}\right), & \text { if } \rho_{m}=-1, \\
O\left(h^{m-3}\right), & \text { if } \rho_{m}=1,\end{cases}
\end{aligned}
$$

as $h \rightarrow 0$, with $N h \leq$ const.

Proof. Using Peano's theorem and Corollary (1.8.2) from Brunner [2004], pp. 43 and 44 , the exact solution of the IAEs system (1) can be obtained as:

$$
\begin{aligned}
& \mathbf{y}\left(t_{n}+\rho h\right)=\sum_{k=1}^{m} \mathbf{Y}_{n k} L_{k}(\rho)+h^{m} \mathbf{R}_{m, n}(\rho), \\
& \mathbf{z}\left(t_{n}+\rho h\right)=\sum_{k=1}^{m} \mathbf{Z}_{n k} L_{k}(\rho)+h^{m} \mathbf{R}_{m, n}^{\prime}(\rho),
\end{aligned}
$$

where $\mathbf{Y}_{n k}=\left\{Y_{l n k}\right\}_{l=1}^{d_{1}}=\mathbf{y}\left(t_{n}+c_{k} h\right), \mathbf{Z}_{n k}=\left\{Z_{l n k}\right\}_{l=1}^{d_{2}}=\mathbf{z}\left(t_{n}+c_{k} h\right)$, and the Peano remainder and kernel are given by

$$
\begin{aligned}
& \mathbf{R}_{m, n}(\rho)=\int_{0}^{1} \mathbf{y}^{(m)}\left(t_{n}+x h\right) K_{m}(\rho, x) d x \\
& \mathbf{R}_{m, n}^{\prime}(\rho)=\int_{0}^{1} \mathbf{z}^{(m)}\left(t_{n}+x h\right) K_{m}(\rho, x) d x,
\end{aligned}
$$

with

$$
K_{m}(\rho, x)=\frac{1}{(m-1)}\left\{(\rho-x)_{+}^{(m-1)}-\sum_{k=1}^{m} L_{k}(\rho)\left(c_{k}-x\right)_{+}^{(m-1)}\right\}, \quad \rho \in(0,1] .
$$

Suppose that $\mathbf{e}=\mathbf{y}-\mathbf{u}$ and $\mathbf{e}^{\prime}=\mathbf{z}-\mathbf{v}$ are the collocation errors, then by considering the relations (8), (4) and (9), (5), we have the following representations

$$
\begin{aligned}
\mathbf{e}\left(t_{n}+\rho h\right) & =\sum_{k=1}^{m} \mathbf{E}_{n k} L_{k}(\rho)+h^{m} \mathbf{R}_{m, n}(\rho), \\
\mathbf{e}^{\prime}\left(t_{n}+\rho h\right) & =\sum_{k=1}^{m} \mathbf{E}_{n k}^{\prime} L_{k}(\rho)+h^{m} \mathbf{R}_{m, n}^{\prime}(\rho),
\end{aligned}
$$

where $\mathbf{E}_{n k}=\mathbf{Y}_{n k}-\mathbf{U}_{n k}$ and $\mathbf{E}_{n k}^{\prime}=\mathbf{Z}_{n k}-\mathbf{V}_{n k}$. 
Subtracting the first and second equation of (1) from (2) and (3), respectively, we obtain

$$
\begin{aligned}
\mathbf{e}(t) & =\int_{0}^{t} \mathbf{K}_{11}(t, s) \mathbf{e}(s) d s+\int_{0}^{t} \mathbf{K}_{12}(t, s) \mathbf{e}^{\prime}(s) d s, \\
\mathbf{0} & =\int_{0}^{t} \mathbf{K}_{21}(t, s) \mathbf{e}(s) d s .
\end{aligned}
$$

Let us set $\rho=\left(s-t_{i}\right) / h,(i=0, \ldots, n)$, then we will have

$$
\begin{aligned}
\mathbf{e}\left(t_{n j}\right)= & h \sum_{i=0}^{n-1} \int_{0}^{1} \mathbf{K}_{11}\left(t_{\left.n j, t_{i}+\rho h\right)} \mathbf{e}\left(t_{i}+\rho h\right) d \rho\right. \\
& +h \sum_{i=0}^{n-1} \int_{0}^{1} \mathbf{K}_{12}\left(t_{n j}, t_{i}+\rho h\right) \mathbf{e}^{\prime}\left(t_{i}+\rho h\right) d \rho \\
& +h \int_{0}^{c_{j}} \mathbf{K}_{11}\left(t_{n j}, t_{n}+\rho h\right) \mathbf{e}\left(t_{n}+\rho h\right) d \rho \\
& +h \int_{0}^{c_{j}} \mathbf{K}_{12}\left(t_{n j}, t_{n}+\rho h\right) \mathbf{e}^{\prime}\left(t_{n}+\rho h\right) d \rho, \\
\mathbf{0}= & h \sum_{i=0}^{n-1} \int_{0}^{1} \mathbf{K}_{21}\left(t_{n j}, t_{i}+\rho h\right) \mathbf{e}\left(t_{i}+\rho h\right) d \rho \\
& +h \int_{0}^{c_{j}} \mathbf{K}_{21}\left(t_{n j}, t_{n}+\rho h\right) \mathbf{e}\left(t_{n}+\rho h\right) d \rho .
\end{aligned}
$$

We now rewrite (15) with $n$ replaced by $n-1$ and $j=m$, subtract this equation from (15) and divide by $h$ :

$$
\begin{aligned}
\int_{0}^{c_{j}} & \mathbf{K}_{21}\left(t_{n j}, t_{n}+\rho h\right) \mathbf{e}\left(t_{n}+\rho h\right) d \rho \\
= & \int_{0}^{c_{m}} \mathbf{K}_{21}\left(t_{n-1, m}, t_{n-1}+\rho h\right) \mathbf{e}\left(t_{n-1}+\rho h\right) d \rho \\
& -\int_{0}^{1} \mathbf{K}_{21}\left(t_{n j}, t_{n-1}+\rho h\right) \mathbf{e}\left(t_{n-1}+\rho h\right) d \rho \\
& -\sum_{i=0}^{n-2} \int_{0}^{1} \mathbf{K}_{21}\left(t_{n j}, t_{i}+\rho h\right)-\mathbf{K}_{21}\left(t_{n-1, m}, t_{i}+\rho h\right) \mathbf{e}\left(t_{i}+\rho h\right) d \rho .
\end{aligned}
$$

In this position, we employ the Taylor series expansion of the components of $\mathbf{K}_{21}$ as follows:

$$
\begin{aligned}
& \mathbf{K}_{21}\left(t_{n j}, t_{i}+\rho h\right)-\mathbf{K}_{21}\left(t_{n-1, m}, t_{i}+\rho h\right) \\
& \quad=c_{j} h \frac{\partial \mathbf{K}_{21}}{\partial t}\left(t_{n}, t_{i}+\rho h\right)+\left(1-c_{m}\right) h \frac{\partial \mathbf{K}_{21}}{\partial t}\left(t_{n}, t_{i}+\rho h\right)+\mathbf{O}(h) .
\end{aligned}
$$

where $\mathbf{O}(h)=\{O(h)\}_{d_{2} \times d_{1}}$. 
We have to distinguish between two cases for $c_{m}$, so let us assume that the domain of $c_{m}$ be divided into two disjoint subsets, $c_{m}<1$ and $c_{m}=1$. We analyze these two cases separately as follows:

\section{Case 1. $c_{m}=1$.}

Inserting (17) into (16), and considering the Taylor series expansion of $\left(\mathbf{K}_{21}\left(t_{n-1, m}, t_{n-1}+\rho h\right)-\mathbf{K}_{21}\left(t_{n j}, t_{n-1}+\rho h\right)\right)$, similar to (17), we can rewrite (16) as:

$$
\int_{0}^{c_{j}} \mathbf{K}_{21}\left(t_{n j}, t_{n}+\rho h\right) \mathbf{e}\left(t_{n}+\rho h\right) d \rho=-\sum_{i=0}^{n-1} \int_{0}^{1} c_{j} h \frac{\partial \mathbf{K}_{21}}{\partial t}\left(t_{n}, t_{i}+\rho h\right) \mathbf{e}\left(t_{i}+\rho h\right) d \rho .
$$

Substituting (10) into (18), yields

$$
\begin{aligned}
\mathbf{0}= & -\sum_{k=1}^{m} \int_{0}^{c_{j}} \mathbf{K}_{21}\left(t_{n j}, t_{n}+\rho h\right) \mathbf{E}_{n k} L_{k}(\rho) d \rho \\
& -\sum_{i=0}^{n-1} \sum_{k=1}^{m} \int_{0}^{1} c_{j} h \frac{\partial \mathbf{K}_{21}}{\partial t}\left(t_{n}, t_{i}+\rho h\right) \mathbf{E}_{i k} L_{k}(\rho) d \rho \\
& -h^{m} \int_{0}^{c_{j}} \mathbf{K}_{21}\left(t_{n j}, t_{n}+\rho h\right) \mathbf{R}_{m, n}(\rho) d \rho \\
& -h^{m} \sum_{i=0}^{n-1} \int_{0}^{1} c_{j} h \frac{\partial \mathbf{K}_{21}}{\partial t}\left(t_{n}, t_{i}+\rho h\right) \mathbf{R}_{m, n}(\rho) d \rho .
\end{aligned}
$$

We also rewrite (14), using (11) and (10) as

$$
\begin{aligned}
\mathbf{E}\left(t_{n j}\right)= & h \sum_{i=0}^{n-1} \sum_{k=1}^{m} \int_{0}^{1} \mathbf{K}_{11}\left(t_{n j}, t_{i}+\rho h\right) \mathbf{E}_{i k} L_{k}(\rho) d \rho \\
& +h \sum_{i=0}^{n-1} \sum_{k=1}^{m} \int_{0}^{1} \mathbf{K}_{12}\left(t_{n j}, t_{i}+\rho h\right) \mathbf{E}_{i k}^{\prime} L_{k}(\rho) d \rho \\
& +h \sum_{k=1}^{m} \int_{0}^{c_{j}} \mathbf{K}_{11}\left(t_{n j}, t_{n}+\rho h\right) \mathbf{E}_{n k} L_{k}(\rho) d \rho \\
& +h \sum_{k=1}^{m} \int_{0}^{c_{j}} \mathbf{K}_{12}\left(t_{n j}, t_{n}+\rho h\right) \mathbf{E}_{n k}^{\prime} L_{k}(\rho) d \rho \\
& +h^{m+1} \sum_{i=0}^{n-1} \int_{0}^{1} \mathbf{K}_{11}\left(t_{n j}, t_{i}+\rho h\right) \mathbf{R}_{m, n}(\rho) d \rho \\
& +h^{m+1} \sum_{i=0}^{n-1} \int_{0}^{1} \mathbf{K}_{12}\left(t_{n j}, t_{i}+\rho h\right) \mathbf{R}_{m, n}^{\prime}(\rho) d \rho
\end{aligned}
$$


On the Convergence Analysis of the Spline Collocation Method for System of Integral Algebraic

$$
\begin{aligned}
& +h^{m+1} \int_{0}^{c_{j}} \mathbf{K}_{11}\left(t_{n j}, t_{n}+\rho h\right) \mathbf{R}_{m, n}(\rho) d \rho \\
& +h^{m+1} \int_{0}^{c_{j}} \mathbf{K}_{12}\left(t_{n j}, t_{n}+\rho h\right) \mathbf{R}_{m, n}^{\prime}(\rho) d \rho .
\end{aligned}
$$

Note that Eqs. (19) and (20) can be written as a compact matrix representation:

$$
\mathbf{A}_{\mathbf{1}} \overline{\mathbf{E}}_{n}=\sum_{i=0}^{n-1} \mathbf{G} \overline{\mathbf{E}}_{i}+\mathbf{C}
$$

where

$$
\begin{gathered}
\overline{\mathbf{E}}_{n}=\left(\begin{array}{c}
\mathbf{E}_{n} \\
\mathbf{E}_{n}^{\prime}
\end{array}\right), \quad \mathbf{E}_{n}=\left\{E_{l, n}\right\}_{l=1}^{d_{1}}=\left(E_{l n 1}, \ldots, E_{l n m}\right)^{T}, \\
\mathbf{E}_{n}^{\prime}=\left\{E_{l, n}^{\prime}\right\}_{l=1}^{d_{2}}=\left(E_{l n 1}^{\prime}, \ldots, E_{l n m}^{\prime}\right)^{T}
\end{gathered}
$$

and

$$
\mathbf{A}_{\mathbf{1}}=\left(\begin{array}{cc}
\mathbf{I}_{m}-h \mathbf{B}_{n}^{(1,1)} & -h \mathbf{B}_{n}^{(1,2)} \\
\mathbf{B}_{n}^{(2,1)} & \{0\}_{d_{2} \times d_{2}}
\end{array}\right)
$$

where $\mathbf{B}_{n}^{(p, l)}=\left(\begin{array}{c}\int_{0}^{c_{j}} \mathbf{K}_{11}\left(t_{n j}, t_{n}+\rho h\right) L_{k}(\rho) d \rho \\ j, k=1, \ldots, m\end{array}\right),(p, l=1,2)$ and $\mathbf{I}_{m}=\operatorname{diag}\left(I_{m}\right)$ is $d_{1} \times d_{1}$ diagonal block matrix,

$$
\begin{aligned}
& \mathbf{G}=\left(\begin{array}{cc}
h \mathbf{B}_{n, i}^{(1,1)} & h \mathbf{B}_{n, i}^{(1,2)} \\
-h c_{j} \tilde{\mathbf{B}}_{n, i}^{(2,1)} & \{0\}_{d_{2} \times d_{2}}
\end{array}\right), \\
& \mathbf{C}=\left(\begin{array}{c}
\left.h^{m+1}\left(\begin{array}{c}
\mathbf{P}_{n}^{(1,1)}+\mathbf{P}_{n}^{\prime(1,2)}+\sum_{i=0}^{n-1} \mathbf{P}_{n, i}^{(1,1)}+\sum_{i=0}^{n-1} \mathbf{P}_{n, i}^{\prime(1,2)}
\end{array}\right)\right), \\
-h^{m} \mathbf{P}_{n}^{(2,1)}-c_{j} h^{m+1} \sum_{i=0}^{n-1} \tilde{\mathbf{P}}_{n, i}^{(2,1)}
\end{array}\right)
\end{aligned}
$$

where the meaning of the matrices $\mathbf{B}_{n, i}^{(p, l)}, \tilde{\mathbf{B}}_{n, i}^{(2,1)}, \mathbf{P}_{n}^{(1,1)}, \ldots$ is clear.

Due to the assumptions of Theorem 1, we have

$$
\left|\operatorname{det}\left(\mathbf{K}_{21}(t, t) \mathbf{K}_{12}(t, t)\right)\right|>0, \quad \forall t \in I,
$$

this shows that $\mathbf{A}_{\mathbf{1}}$ is an invertible matrix with the following representation: [see Bernstein (2005), pp. 44]:

$$
\mathbf{A}_{1}^{-1}=\left(\begin{array}{cc}
F_{1}^{-1}+F_{1}^{-1} F_{2}\left(F_{4}-F_{3} F_{1}^{-1} F_{2}\right)^{-1} F_{3} F_{1}^{-1} & -F_{1}^{-1} F_{2}\left(F_{4}-F_{3} F_{1}^{-1} F_{2}\right)^{-1} \\
-\left(F_{4}-F_{3} F_{1}^{-1} F_{2}\right)^{-1} F_{3} F_{1}^{-1} & \left(F_{4}-F_{3} F_{1}^{-1} F_{2}\right)^{-1}
\end{array}\right),
$$

where $F_{1}=\mathbf{I}_{m}-h \mathbf{B}_{n}^{(1,1)}, F_{2}=-h \mathbf{B}_{n}^{(1,2)}, F_{3}=\mathbf{B}_{n}^{(2,1)}$ and $F_{4}=\{0\}_{d_{2} \times d_{2}}$. 
With these notations, $\mathbf{A}_{\mathbf{1}}^{-\mathbf{1}}$ is unbounded if $h$ is sufficiently small. Multiplying (21) by $\mathbf{A}_{\mathbf{1}}^{-\mathbf{1}}$, the block matrices $\mathbf{A}_{\mathbf{1}}^{-\mathbf{1}} \mathbf{G}$ and $\mathbf{A}_{1}^{-1} \mathbf{C}$ may be obtained as:

$$
\mathbf{A}_{\mathbf{1}}^{-\mathbf{1}} \mathbf{G}=\left(\begin{array}{cc}
\{0\}_{d_{1} \times d_{1}} & \{0\}_{d_{1} \times d_{2}} \\
\mathbf{D}_{\mathbf{1}} & \mathbf{D}_{\mathbf{2}}
\end{array}\right), \quad \mathbf{A}_{\mathbf{1}}^{-\mathbf{1}} \mathbf{C}=\left(\begin{array}{c}
\left\{O\left(h^{m}\right)\right\}_{d_{1} \times 1} \\
\left\{O\left(h^{m-1}\right)\right\}_{d_{2} \times 1}
\end{array}\right),
$$

where

$$
\begin{aligned}
& \mathbf{D}_{1}=-h\left(F_{4}-F_{3} F_{1}^{-1} F_{2}\right)^{-1} F_{3} F_{1}^{-1} \mathbf{B}_{n, i}^{(1,1)}-h c_{j}\left(F_{4}-F_{3} F_{1}^{-1} F_{2}\right)^{-1} \tilde{\mathbf{B}}_{n, i}^{(2,1)}, \\
& \mathbf{D}_{\mathbf{2}}=-h\left(F_{4}-F_{3} F_{1}^{-1} F_{2}\right)^{-1} F_{3} F_{1}^{-1} \mathbf{B}_{n, i}^{(1,2)},
\end{aligned}
$$

in which $h \rightarrow 0$, with $n h=T \leq$ const.

Considering the above representations, we conclude

$$
\left\|\mathbf{E}_{n}\right\|_{1}=O\left(h^{m}\right),
$$

where $\|\cdot\|_{1}$ denotes the matrix (operator) norm induced by the $L^{1}$-norm, and also

$$
\mathbf{E}_{n}^{\prime}=\sum_{i=0}^{n-1} \mathbf{D}_{\mathbf{2}} \mathbf{E}_{i}^{\prime}+\sum_{i=0}^{n-1} \mathbf{D}_{\mathbf{1}} \mathbf{E}_{i}+\mathbf{O}\left(h^{m-1}\right),
$$

where $\mathbf{O}\left(h^{m-1}\right)=\left\{O\left(h^{m-1}\right)\right\}_{d_{2} \times 1}$. So, Eq. (22) can be written as

$$
\mathbf{E}_{n}^{\prime}=\sum_{i=0}^{n-1} \mathbf{D}_{2} \mathbf{E}_{i}^{\prime}+\mathbf{O}\left(h^{m-1}\right)
$$

It then follows from the Gronwall's inequality [Brunner (2004)]

$$
\left\|\mathbf{E}_{n}^{\prime}\right\|_{1}=O\left(h^{m-1}\right),
$$

therefore, the following estimates for (11) and (10) can be obtained

$$
\begin{gathered}
\left|\mathbf{e}\left(t_{n}+\rho h\right)\right| \leq \Omega_{m}\left\|\mathbf{E}_{n}\right\|_{1}+h^{m} M_{m} K_{m}, \\
\left|\mathbf{e}^{\prime}\left(t_{n}+\rho h\right)\right| \leq \Omega_{m}\left\|\mathbf{E}_{n}^{\prime}\right\|_{1}+h^{m} M_{m}^{\prime} K_{m},
\end{gathered}
$$

where

$$
M_{m}=\left\|\mathbf{y}^{(m)}\right\|_{\infty}, \quad M_{m}^{\prime}=\left\|\mathbf{z}^{(m)}\right\|_{\infty}, \quad K_{m}=\max _{\rho \in(0,1]} \int_{0}^{1}\left|K_{m}(\rho, x)\right| d x,
$$

and

$$
\Omega_{m}=\max _{j}\left\|L_{j}\right\|_{\infty} .
$$

Now, using (25) and (26) for $c_{m}=1$, we get

$$
\|\mathbf{y}-\mathbf{u}\|_{\infty}=O\left(h^{m}\right), \quad\|\mathbf{z}-\mathbf{v}\|_{\infty}=O\left(h^{m-1}\right) .
$$

Case 2. $c_{m}<1$.

In this case, we will make use of the result of Brunner [2004] which gives the conditions and global order of convergence of the spline collocation method for 
the first kind integral equations. Actually, as stated in Theorem 2.4.2 of Brunner [2004], p. 123, we note that the second equation of (1) is a first kind Volterra integral equation with solution $y \in C(I)$. It can be seen that the functions $g$ and $K_{21}$ of (1) are satisfy to the hypothesis of the theorem

$$
g \in C^{1}(I), \quad g(0)=0, \quad K_{21} \in C^{1}(D), \quad\left|\operatorname{det}\left(K_{21}(t, t)\right)\right| \geq k 0>0, \quad t \in I,
$$

hence there exists an $h>0$, such that for all meshes $I_{h}$, with diameter $h \in(0, \bar{h})$, the corresponding linear algebraic system arises from the spline collocation equation, possesses a unique solution and the collocation solution $\mathbf{u}$ converges to the exact solution $\mathbf{y}$, with the error estimation:

$$
\|\mathbf{y}-\mathbf{u}\|_{\infty}= \begin{cases}O\left(h^{m}\right), & \text { if } \rho_{m} \in[-1,1), \\ O\left(h^{m-1}\right), & \text { if } \rho_{m}=1 .\end{cases}
$$

Actually, the convergence properties of $\mathbf{y}$ to $\mathbf{u}$, in this case may be discussed separately from $\mathbf{z}$. So, our claim is obtaining the attainable order of convergence of $\|\mathbf{z}-\mathbf{v}\|_{\infty}$.

Rewriting Eq. (16), after inserting (17) and (10) get:

$$
\begin{aligned}
\mathbf{0}= & \sum_{k=1}^{m} \int_{0}^{c_{j}} \mathbf{K}_{21}\left(t_{n j}, t_{n}+\rho h\right) \mathbf{E}_{n k} L_{k}(\rho) d \rho \\
& +\sum_{k=1}^{m} \int_{c_{m}}^{1} \mathbf{K}_{21}\left(t_{(n-1) m}, t_{n-1}+\rho h\right) \mathbf{E}_{(n-1) k} L_{k}(\rho) d \rho \\
& +\sum_{i=0}^{n-1} \sum_{k=1}^{m} \int_{0}^{1}\left(c_{j} h+\left(1-c_{m}\right) h\right) \frac{\partial \mathbf{K}_{21}}{\partial t}\left(t_{n}, t_{i}+\rho h\right) \mathbf{E}_{i k} L_{k}(\rho) d \rho \\
& +h^{m} \int_{0}^{c_{j}} \mathbf{K}_{21}\left(t_{n j}, t_{n}+\rho h\right) \mathbf{R}_{m, n}(\rho) d \rho \\
& +h^{m} \int_{c_{m}}^{1} \mathbf{K}_{21}\left(t_{(n-1) m}, t_{n-1}+\rho h\right) \mathbf{R}_{m, n}(\rho) d \rho \\
& +h^{m} \sum_{i=0}^{n-1} \int_{0}^{1}\left(c_{j} h+\left(1-c_{m}\right) h\right) \frac{\partial \mathbf{K}_{21}}{\partial t}\left(t_{n}, t_{i}+\rho h\right) \mathbf{R}_{m, n}(\rho) d \rho .
\end{aligned}
$$

Equations (20) and (27) can be rewritten in the matrix notation

$$
\mathbf{A}_{\mathbf{1}} \overline{\mathbf{E}}_{n}=\mathrm{Q} \overline{\mathbf{E}}_{n-1}+\sum_{i=0}^{n-1} \mathbf{G}^{\prime} \overline{\mathbf{E}}_{i}+\mathbf{C}^{\prime}
$$

where

$$
\mathbf{Q}=\left(\begin{array}{cc}
\{0\}_{d_{1} \times d_{1}} & \{0\}_{d_{1} \times d_{2}} \\
\mathbf{Q}_{\mathbf{0}} & \{0\}_{d_{2} \times d_{2}}
\end{array}\right), \quad \mathbf{Q}_{\mathbf{0}}=\left(\begin{array}{c}
-\int_{c_{m}}^{1} \mathbf{K}_{21}\left(t_{(n-1) m}, t_{n-1}+\rho h\right) L_{k}(\rho) d \rho \\
k=1, \ldots, m
\end{array}\right),
$$


F. Ghoreishi, M. Hadizadeh \& S. Pishbin

$$
\begin{gathered}
\mathbf{G}^{\prime}=\left(\begin{array}{cc}
h \mathbf{B}_{n, i}^{(1,1)} & h \mathbf{B}_{n, i}^{(1,2)} \\
-\left(c_{j}+\left(1-c_{m}\right)\right) h \tilde{\mathbf{B}}_{n, i}^{(2,1)} & \{0\}_{d_{2} \times d_{2}}
\end{array}\right), \\
\mathbf{C}^{\prime}=\left(\begin{array}{c}
h^{m+1}\left(\mathbf{P}_{n}^{(1,1)}+\mathbf{P}_{n}^{\prime(1,2)}+\sum_{i=0}^{n-1} \mathbf{P}_{n, i}^{(1,1)}+\sum_{i=0}^{n-1} \mathbf{P}_{n, i}^{\prime(1,2)}\right) \\
h^{m}\left(\mathbf{Q}_{\mathbf{1}}-\mathbf{P}_{n}^{(2,1)}\right)-c_{j} h^{m+1} \sum_{i=0}^{n-1} \tilde{\mathbf{P}}_{n, i}^{(2,1)}
\end{array}\right),
\end{gathered}
$$

and $\mathbf{Q}_{\mathbf{1}}=\int_{c_{m}}^{1} \mathbf{K}_{21}\left(t_{(n-1) m}, t_{n-1}+\rho h\right) \mathbf{R}_{m, n}(\rho) d \rho$.

Multiplying (28) by $\mathbf{A}_{\mathbf{1}}^{-\mathbf{1}}$, the second equation of the resulting system is obtained as follows:

$$
\mathbf{E}_{n}^{\prime}=\mathbf{D}_{\mathbf{2}} \mathbf{E}_{n-1}^{\prime}+\sum_{i=0}^{n-2} \mathbf{D}_{\mathbf{2}} \mathbf{E}_{i}^{\prime}+\mathbf{K} \mathbf{Q}_{\mathbf{0}} \mathbf{E}_{n-1}+\sum_{i=0}^{n-1} \mathbf{D}_{\mathbf{1}}^{\prime} \mathbf{E}_{i}+\mathbf{O}\left(h^{m-1}\right),
$$

where $\mathbf{K}=\left(F_{4}-F_{3} F_{1}^{-1} F_{2}\right)^{-1}$.

Consequently, $\mathbf{B}_{n, i}^{(1,2)}$ may be written as:

$$
\mathbf{B}_{n, i}^{(1,2)}=\left(\begin{array}{c}
\int_{0}^{c_{m}} \mathbf{K}_{12}\left(t_{n j}, t_{i}+\rho h\right) L_{k}(\rho) d \rho+\int_{c_{m}}^{1} \mathbf{K}_{12}\left(t_{n j}, t_{i}+\rho h\right) L_{k}(\rho) d \rho \\
k=1, \ldots, m
\end{array}\right) .
$$

From this, we can write $\mathbf{D}_{\mathbf{2}}=\mathbf{D}_{\mathbf{3}}+\mathbf{D}_{\mathbf{4}}$, such that

$$
\begin{aligned}
& \mathbf{D}_{3}=-h\left(F_{4}-F_{3} F_{1}^{-1} F_{2}\right)^{-1} F_{3} F_{1}^{-1}\left(\int_{0}^{c_{m}} \mathbf{K}_{12}\left(t_{n j}, t_{i}+\rho h\right) L_{k}(\rho) d \rho\right), \\
& \left.\mathbf{D}_{4}=-h\left(F_{4}-F_{3} F_{1}^{-1} F_{2}\right)^{-1} F_{3} F_{1}^{-1}\left(\int_{c_{m}}^{1} \mathbf{K}_{12}\left(t_{n j}, t_{i}+\rho h\right) L_{k}(\rho) d \rho\right)\right) .
\end{aligned}
$$

Using (30) and (31), Eq. (29) can be written as:

$$
\mathbf{E}_{n}^{\prime}=\left(\mathbf{D}_{\mathbf{4}}+\mathbf{D}_{\mathbf{3}}\right) \mathbf{E}_{n-1}^{\prime}+\sum_{i=0}^{n-2} \mathbf{D}_{\mathbf{2}} \mathbf{E}_{i}^{\prime}+\mathbf{K} \mathbf{Q}_{\mathbf{0}} \mathbf{E}_{n-1}+\sum_{i=0}^{n-1} \mathbf{D}_{\mathbf{1}}^{\prime} \mathbf{E}_{i}+\mathbf{O}\left(h^{m-1}\right)
$$

Now, we first rewrite $\mathbf{B}_{n}^{(1,2)}$ as

$$
\mathbf{B}_{n}^{(1,2)}=\int_{0}^{c_{j}} \mathbf{K}_{12}\left(t_{n j}, t_{n}+\rho h\right) L_{k}(\rho) d \rho=\mathbf{K}_{12}\left(t_{n}, t_{n}\right) \bigotimes A+\{O(h)\}_{d_{1} \times d_{2}},
$$

where $A=\left(\begin{array}{c}\int_{0}^{c_{j}} L_{k}(\rho) d \rho \\ k, j=1, \ldots, m\end{array}\right)$ and $\bigotimes$ is Kronecker product. Also, we have

$$
\int_{c_{m}}^{1} \mathbf{K}_{12}\left(t_{n j}, t_{i}+\rho h\right) L_{k}(\rho) d \rho=\mathbf{K}_{12}\left(t_{i}, t_{i}\right) \bigotimes F+\{O(h)\}_{d_{1} \times d_{2}},
$$


On the Convergence Analysis of the Spline Collocation Method for System of Integral Algebraic

where $F=\left(\begin{array}{l}\int_{c m}^{1} L_{k}(\rho) d \rho \\ k=1, \ldots, m\end{array}\right)$. On the other hand

$$
\begin{aligned}
\mathbf{D}_{4}= & -\left(F_{3} F_{1}^{-1}\left(\mathbf{K}_{12}\left(t_{n}, t_{n}\right) \bigotimes A+\{O(h)\}_{d_{1} \times d_{2}}\right)\right)^{-1} F_{3} F_{1}^{-1} \\
& \times\left(\mathbf{K}_{12}\left(t_{i}, t_{i}\right) \bigotimes F+\{O(h)\}_{d_{1} \times d_{2}}\right) .
\end{aligned}
$$

In order to describe the key ideas without having to resort to complex notation for $\mathbf{D}_{4}$, using properties of the Kronecker product we obtain

$$
\mathbf{D}_{4} \simeq \mathbf{D}_{5} \bigotimes A^{-1} F
$$

where $\mathbf{D}_{5}$ is an identity $d_{2} \times d_{2}$ matrix. According to Lemma 2.4.3 of Brunner [2004], we know that $A^{-1} F$ has a nontrivial eigenvalue as

$$
\lambda=\rho_{m}=(-1)^{m} \prod_{i=1}^{m} \frac{1-c_{i}}{c_{i}} .
$$

It follows from the elementary theory of the difference equations [see Elaydi (1999)] that the solutions of the system of the first-order difference equations (32) remain uniformly bounded, if and only if $|\lambda|=\left|\rho_{m}\right| \leq 1$. [For further details see Brunner (2004), p. 126.]

Now, if $\rho_{m}=[-1,1)$ then by considering Theorem 2.4.2 from Brunner [2004], we conclude $\left\|\mathbf{E}_{n}\right\|_{1}=O\left(h^{m}\right),(n=0, \ldots, N-1)$ so, Eq. (32) can be written as:

$$
\mathbf{E}_{n}^{\prime}=\left(\mathbf{D}_{\mathbf{4}}+\mathbf{D}_{\mathbf{3}}\right) \mathbf{E}_{n-1}^{\prime}+\sum_{i=0}^{n-2} \mathbf{D}_{\mathbf{2}} \mathbf{E}_{i}^{\prime}+\mathbf{O}\left(h^{m-1}\right) \text {. }
$$

Since $A^{-1} F$ is diagonalizable, there exists a nonsingular matrix $P$ such that $\mathbf{D}_{4} \simeq \mathbf{D}_{5} \otimes P D_{\lambda} P^{-1}$ where $D_{\lambda}=\operatorname{diag}(\lambda, 0, \ldots, 0)$. Multiplying $(33)$ by $\widehat{\mathbf{P}}_{d_{2} \times d_{2}}=$ $\operatorname{diag}\left(P^{-1}, P^{-1}, \ldots, P^{-1}\right)$ and defining $\mathbf{Z}_{n}=\widehat{\mathbf{P}} \mathbf{E}_{n}^{\prime}$, we obtain

$$
\mathbf{Z}_{n}=\left(\mathbf{D}_{5} \bigotimes D_{\lambda}\right) \mathbf{Z}_{n-1}+\widehat{\mathbf{P}} \mathbf{D}_{\mathbf{3}} \widehat{\mathbf{P}}^{-1} \mathbf{Z}_{n-1}+\sum_{i=0}^{n-2} \widehat{\mathbf{P}} \mathbf{D}_{\mathbf{2}} \widehat{\mathbf{P}}^{-1} \mathbf{Z}_{i}+\mathbf{O}\left(h^{m-1}\right)
$$

Taking norms, we arrive at inequality of the form

$$
\left\|\mathbf{Z}_{n}\right\| \leq|\lambda|\left\|\mathbf{Z}_{n-1}\right\|+\gamma_{3}\left\|\mathbf{Z}_{n-1}\right\|+\gamma_{2} \sum_{i=0}^{n-2}\left\|\mathbf{Z}_{i}\right\|+O\left(h^{m-1}\right) .
$$

Repeated insertion of this formula and considering the Lemma 6 of Hairer et al. [1983] give

$$
\left\|\mathbf{Z}_{n}\right\| \leq|\lambda|^{n}\left\|\mathbf{Z}_{0}\right\|+\sum_{i=1}^{n}|\lambda|^{n-i} O\left(h^{m-1}\right)+O\left(h^{m-1}\right) .
$$

If $|\lambda|<1$, then $\left\|\mathbf{Z}_{n}\right\|=O\left(h^{m-1}\right)$, and

$$
\left\|\mathbf{E}_{n}^{\prime}\right\|_{1}=O\left(h^{m-1}\right) .
$$


If $|\lambda|=1$, then $\left\|\mathbf{Z}_{n}\right\|=O\left(h^{m-2}\right)$, and

$$
\left\|\mathbf{E}_{n}^{\prime}\right\|_{1}=O\left(h^{m-2}\right) .
$$

In the case of $\rho_{m}=1$, by considering Theorem 2.4.2 from Brunner [2004], we have $\left\|\mathbf{E}_{n}\right\|=O\left(h^{m-1}\right),(n=0, \ldots, N-1)$ and Eq. (32) can be written as:

$$
\mathbf{E}_{n}^{\prime}=\left(\mathbf{D}_{\mathbf{4}}+\mathbf{D}_{\mathbf{3}}\right) \mathbf{E}_{n-1}^{\prime}+\sum_{i=0}^{n-2} \mathbf{D}_{\mathbf{2}} \mathbf{E}_{i}^{\prime}+\mathbf{O}\left(h^{m-2}\right) .
$$

Using a similar procedure as outlined for (34)-(36), the following result can be obtained for the relation (39)

$$
\left\|\mathbf{E}_{n}^{\prime}\right\|_{1}=O\left(h^{m-3}\right) .
$$

Now, (37) and (26) for $c_{m}<1$ and $\rho_{m}=(-1,1)$, give

$$
\|\mathbf{z}-\mathbf{v}\|_{\infty}=O\left(h^{m-1}\right) .
$$

Also, (38) and (26) for $c_{m}<1$ and $\rho_{m}=-1$ ( $m$ is odd), yield

$$
\|\mathbf{z}-\mathbf{v}\|_{\infty}=O\left(h^{m-2}\right) .
$$

Finally, using (40) and (26) for $c_{m}<1$, and $\rho_{m}=1$ ( $m$ is even), we conclude

$$
\|\mathbf{z}-\mathbf{v}\|_{\infty}=O\left(h^{m-3}\right),
$$

which leads to the stated estimates of the theorem.

We must emphasize that, the convergence orders in Theorem 2 are completely different from the analogous one for index-1 IAEs in Kauthen [2000]. It is interesting to note that for the index-2 IAEs $(1)$ in the case of $c_{m}<1$, the orders of convergence $O\left(h^{m-2}\right)$ and $O\left(h^{m-3}\right)$ are attained for $z$-component, if $m$ is odd $\left(\rho_{m}=-1\right)$ and even $\left(\rho_{m}=1\right)$, respectively. However, following Kauthen [2000], for IAEs of index-1 the global convergence orders $O\left(h^{m}\right)$ and $O\left(h^{m-1}\right)$ for $z$ have been obtained while $-1 \leq \rho_{m}<1$ and $\rho_{m}=1$, respectively.

\section{Numerical Results and Discussions}

To illustrate the theoretical findings of the preceding sections, we will present some numerical results of the proposed method for the considered IAEs systems of index2 from Hadizadeh et al. [2011]. Let $\mathbf{u}=\left(u_{1}, \ldots, u_{d_{1}}\right)^{T}$ and $\mathbf{v}=\left(v_{1}, \ldots, v_{d_{2}}\right)^{T}$ with $\left\{u_{l}\right\}_{l=1}^{d_{1}},\left\{v_{l}\right\}_{l=1}^{d_{2}} \in S_{m-1}^{-1}\left(\Pi_{N}\right)$ be the collocation solution. We consider the collocation parameters in two cases, $c_{m}=1$ and $c_{m}<1$. For $c_{m}=1$, we choose the Radau II points (i.e., the zeros of $\left.P_{m-1}(2 s-1)-P_{m}(2 s-1)\right)$ as collocation parameters and for the case $c_{m}<1$, we apply the zeros of $P_{m}(2 s-1)$ as the collocation parameters in which $P_{m}$ denotes the Legendre polynomial of degree $m$. The accuracy of the proposed method is measured by computing the difference 
between the exact and the numerical solution at each gridpoints. We also report the observed order of convergence from the maximum errors $\|\mathbf{y}-\mathbf{u}\|_{\infty}$ and $\|\mathbf{z}-\mathbf{v}\|_{\infty}$ at the gridpoints. All the calculations were supported by the Mathematica ${ }^{\circledR}$.

Example 1 [From Hadizadeh et al. (2011)]. Consider the following system of linear semi-explicit index-2 IAEs of the Hessenberg form:

$$
A X(t)=G(t)+\int_{0}^{t} K(t, s) X(s) d s, \quad t \in[0,1],
$$

where

$$
\begin{gathered}
A=\left(\begin{array}{ll}
1 & 0 \\
0 & 0
\end{array}\right), \quad K(t, s)=\left(\begin{array}{cc}
e^{t+s} & (s+1)^{2} \\
s+t+2 & 0
\end{array}\right), \\
X(t)=(y(t), z(t))^{T}, \quad G(t)=(f(t), g(t))^{T},
\end{gathered}
$$

and

$$
\begin{aligned}
f(t)= & \sin t-\frac{1}{2} e^{t}\left(1+e^{t}(-\cos t+\sin t)\right) \\
& -\frac{1}{4}\left(-2+2(1+t) \cos 2 t+\left(1+4 t+2 t^{2}\right) \sin 2 t\right), \\
g(t)= & -(2+t)+2(1+t) \cos t-\sin t .
\end{aligned}
$$

The exact solution of the system is:

$$
y(t)=\sin t, \quad z(t)=\cos 2 t .
$$

The maximum errors have been shown for different values of $m$ and $N$ at the gridpoints in the Tables 1, 2, 5, and 6. Also, the orders of convergence from the maximum errors at the gridpoints have been reported in Tables 3, 4, 7, and 8 which they confirm the theoretical results of the Theorem 2.

Example 2 [From Hadizadeh et al. (2011)].

$$
A X(t)=G(t)+\int_{0}^{t} K(t, s) X(s) d s, \quad t \in[0,1],
$$

Table 1. Maximum errors $\|y-u\|_{\infty}$ and $\|z-v\|_{\infty}$ with $c_{m}=1$ in Example 1.

\begin{tabular}{cccccccc}
\hline & \multicolumn{3}{c}{$\|y-u\|_{\infty}$} & & \multicolumn{3}{c}{$\|z-v\|_{\infty}$} \\
\cline { 2 - 3 } \cline { 6 - 7 }$m$ & $N=16$ & $N=32$ & $N=64$ & & $N=16$ & $N=32$ & $N=64$ \\
\hline 2 & $4.00 \mathrm{E}-4$ & $1.04 \mathrm{E}-4$ & $2.65 \mathrm{E}-5$ & & $2.69 \mathrm{E}-2$ & $1.68 \mathrm{E}-2$ & $9.38 \mathrm{E}-3$ \\
3 & $4.11 \mathrm{E}-6$ & $5.16 \mathrm{E}-7$ & $6.46 \mathrm{E}-8$ & & $4.63 \mathrm{E}-4$ & $1.13 \mathrm{E}-4$ & $2.78 \mathrm{E}-5$ \\
4 & $2.73 \mathrm{E}-8$ & $1.76 \mathrm{E}-9$ & $1.11 \mathrm{E}-10$ & & $6.58 \mathrm{E}-6$ & $9.82 \mathrm{E}-7$ & $1.33 \mathrm{E}-7$ \\
\hline
\end{tabular}


Table 2. Maximum errors $\|y-u\|_{\infty}$ and $\|z-v\|_{\infty}$ with $c_{m}<1$ in Example 1 .

\begin{tabular}{cccccccc}
\hline & \multicolumn{3}{c}{$\|y-u\|_{\infty}$} & & \multicolumn{3}{c}{$\|z-v\|_{\infty}$} \\
\cline { 2 - 4 }$m$ & $N=16$ & $N=32$ & $N=64$ & & $N=16$ & $N=32$ & $N=64$ \\
\hline 3 & $6.75 \mathrm{E}-6$ & $8.38 \mathrm{E}-7$ & $1.04 \mathrm{E}-7$ & & $4.10 \mathrm{E}-3$ & $2.00 \mathrm{E}-3$ & $9.95 \mathrm{E}-4$ \\
4 & $3.69 \mathrm{E}-7$ & $4.65 \mathrm{E}-8$ & $5.83 \mathrm{E}-9$ & & $7.24 \mathrm{E}-4$ & $3.65 \mathrm{E}-4$ & $1.82 \mathrm{E}-4$ \\
\hline
\end{tabular}

Table 3. Orders of convergence of $u$ and $v$ with $c_{m}=1$ in Example 1.

\begin{tabular}{cccccccc}
\hline & \multicolumn{3}{c}{$u$} & & \multicolumn{3}{c}{$v$} \\
\cline { 2 - 3 } \cline { 6 - 7 }$m$ & $N=16$ & $N=32$ & $N=64$ & & $N=16$ & $N=32$ & $N=64$ \\
\hline 2 & 1.87 & 1.96 & 1.97 & & 0.36 & 0.67 & 0.84 \\
3 & 2.98 & 2.99 & 2.99 & & 2.05 & 2.03 & 2.02 \\
4 & 3.90 & 3.95 & 3.98 & & 2.79 & 2.79 & 2.88 \\
\hline
\end{tabular}

Table 4. Orders of convergence of $u$ and $v$ with $c_{m}<1, \rho_{m}=-1$ and $\rho_{m}=1$ in Example 1 .

\begin{tabular}{llcccccc}
\hline & \multicolumn{3}{c}{$u$} & & \multicolumn{3}{c}{$v$} \\
\cline { 2 - 3 } \cline { 6 - 7 }$m$ & $N=16$ & $N=32$ & $N=64$ & & $N=16$ & $N=32$ & $N=64$ \\
\hline 3 & 3.02 & 3.00 & 3.00 & & 1.10 & 1.03 & 1.00 \\
4 & 2.97 & 2.98 & 2.99 & & 0.94 & 0.98 & 1.00 \\
\hline
\end{tabular}

Table 5. Maximum errors $\left\|y_{1}-u_{1}\right\|_{\infty},\left\|y_{2}-u_{2}\right\|_{\infty}$ and $\left\|z_{1}-v_{1}\right\|_{\infty}$ with $c_{m}=1$ in Example 2.

\begin{tabular}{|c|c|c|c|c|c|c|c|c|}
\hline \multirow[b]{2}{*}{$m$} & \multicolumn{2}{|l|}{$\left\|y_{1}-u_{1}\right\|_{\infty}$} & \multicolumn{3}{|c|}{$\left\|y_{2}-u_{2}\right\|_{\infty}$} & \multicolumn{3}{|c|}{$\left\|z_{1}-v_{1}\right\|_{\infty}$} \\
\hline & $N=16$ & $N=64$ & $N=16$ & $N=32$ & $N=64$ & $N=16$ & $N=32$ & $N=64$ \\
\hline 2 & $6.08 \mathrm{E}-31.54 \mathrm{E}-3$ & $3.89 \mathrm{E}-4$ & $3.08 \mathrm{E}-3$ & $7.79 \mathrm{E}-4$ & $1.95 \mathrm{E}-4$ & $2.79 \mathrm{E}-1$ & $1.45 \mathrm{E}-1$ & $7.42 \mathrm{E}-2$ \\
\hline 3 & $7.93 \mathrm{E}-51.01 \mathrm{E}-5$ & $1.28 \mathrm{E}-6$ & $3.96 \mathrm{E}-5$ & $5.07 \mathrm{E}-6$ & $6.40 \mathrm{E}-7$ & $3.82 \mathrm{E}-3$ & $9.75 \mathrm{E}-4$ & $2.46 \mathrm{E}-4$ \\
\hline 4 & $6.06 \mathrm{E}-73.86 \mathrm{E}-8$ & $2.44 \mathrm{E}-9$ & $3.03 \mathrm{E}-7$ & $1.93 \mathrm{E}-8$ & $1.22 \mathrm{E}-9$ & $9.70 \mathrm{E}-5$ & $1.23 \mathrm{E}-5$ & $1.56 \mathrm{E}-6$ \\
\hline
\end{tabular}

Table 6. Maximum errors $\left\|y_{1}-u_{1}\right\|_{\infty},\left\|y_{2}-u_{2}\right\|_{\infty}$ and $\left\|z_{1}-v_{1}\right\|_{\infty}$ with $c_{m}<1$ in Example 2.

\begin{tabular}{|c|c|c|c|c|c|c|c|c|}
\hline \multirow[b]{2}{*}{$m$} & \multicolumn{2}{|l|}{$\left\|y_{1}-u_{1}\right\|_{\infty}$} & \multicolumn{3}{|c|}{$\left\|y_{2}-u_{2}\right\|_{\infty}$} & \multicolumn{3}{|c|}{$\left\|z_{1}-v_{1}\right\|_{\infty}$} \\
\hline & $N=32$ & $N=64$ & $N=16$ & $N=32$ & $N=64$ & $N=16$ & $N=32$ & $N=64$ \\
\hline 3 & $7.93 \mathrm{E}-51.04 \mathrm{E}-5$ & $1.33 \mathrm{E}-6$ & $4.89 \mathrm{E}-5$ & $6.76 \mathrm{E}-6$ & $8.89 \mathrm{E}-7$ & $8.15 \mathrm{E}-2$ & $4.08 \mathrm{E}-2$ & $2.04 \mathrm{E}-2$ \\
\hline 4 & $1.93 \mathrm{E}-52.47 \mathrm{E}-6$ & $3.13 \mathrm{E}-7$ & $1.27 \mathrm{E}-5$ & $1.65 \mathrm{E}-6$ & $2.10 \mathrm{E}-7$ & $3.47 \mathrm{E}-2$ & $1.78 \mathrm{E}-2$ & $9.06 \mathrm{E}-3$ \\
\hline
\end{tabular}


On the Convergence Analysis of the Spline Collocation Method for System of Integral Algebraic

Table 7 . Orders of convergence of $u_{1}, u_{2}$, and $v_{1}$ with $c_{m}=1$ in Example 2 .

\begin{tabular}{|c|c|c|c|c|c|c|c|c|c|}
\hline \multirow[b]{2}{*}{$m$} & \multicolumn{3}{|c|}{$u_{1}$} & \multicolumn{3}{|c|}{$u_{2}$} & \multicolumn{3}{|c|}{$v_{1}$} \\
\hline & $N=16$ & $N=32$ & $N=64$ & $N=16$ & $N=32$ & $N=64$ & $N=16$ & $N=32$ & $N=64$ \\
\hline 2 & 1.94 & 1.98 & 1.98 & 1.96 & 1.98 & 1.99 & 0.88 & 0.94 & 0.96 \\
\hline 3 & 2.93 & 2.97 & 2.98 & 2.93 & 2.96 & 2.98 & 1.94 & 1.97 & 1.98 \\
\hline 4 & 3.94 & 3.97 & 3.98 & 3.93 & 3.97 & 3.98 & 2.93 & 2.97 & 2.98 \\
\hline
\end{tabular}

Table 8. Orders of convergence of $u_{1}, u_{2}$, and $v_{1}$ with $c_{m}<1, \rho_{m}=-1$ and $\rho_{m}=1$ in Example 2 .

\begin{tabular}{|c|c|c|c|c|c|c|c|c|c|}
\hline \multirow[b]{2}{*}{$m$} & \multicolumn{3}{|c|}{$u_{1}$} & \multicolumn{3}{|c|}{$u_{2}$} & \multicolumn{3}{|c|}{$v_{1}$} \\
\hline & $N=16$ & $N=32$ & $N=64$ & $N=16$ & $N=32$ & $N=64$ & $N=16$ & $N=32$ & $N=64$ \\
\hline 3 & 2.86 & 2.93 & 2.96 & 2.73 & 2.85 & 2.92 & 0.99 & 0.99 & 1 \\
\hline 4 & 2.93 & 2.96 & 2.98 & 2.90 & 2.94 & 2.97 & 0.92 & 0.96 & 0.97 \\
\hline
\end{tabular}

where

$$
\begin{gathered}
A=\left(\begin{array}{lll}
1 & 0 & 0 \\
0 & 1 & 0 \\
0 & 0 & 0
\end{array}\right), \quad K(t, s)=\left(\begin{array}{ccc}
\frac{3-2 s}{2-s} & \frac{3-s}{2-s} & 2(2-s) \\
\frac{-1}{s-2} & -1 & 1 \\
s+2 & s^{2}-4 & 0
\end{array}\right), \\
X(t)=\left(y_{1}(t), y_{2}(t), z_{1}(t)\right)^{T}, \quad G(t)=\left(1,2 e^{t}-1,-1+e^{t}\left(-t^{2}+t+1\right)\right)^{T},
\end{gathered}
$$

with the exact solution:

$$
y_{1}(t)=y_{2}(t)=e^{t}, \quad z_{1}(t)=-\frac{e^{t}}{2-t} .
$$

We compare the results obtained by the presented method with those obtained by Jacobi spectral method in Hadizadeh et al. [2011]. However, we note that, spectral methods are global methods such that the computation at any given point depends not only on information at neighboring points, but on information from the entire domain. Due to completely smoothness of the exact solutions for two previous examples, the results obtained using Jacobi spectral method Hadizadeh et al. [2011] is more accurate than the spline collocation method in this case. The error behaviors obtained by the spline collocation and Jacobi spectral methods for the special values of $m$ and $N$ in Examples 1 and 2 are shown in Figs. 1 and 2, respectively.

In this position, for the better comparison purpose, we present the following test problem:

Example 3. Consider the following IAEs system:

$$
A X(t)=G(t)+\int_{0}^{t} K(t, s) X(s) d s, \quad t \in[0,1],
$$



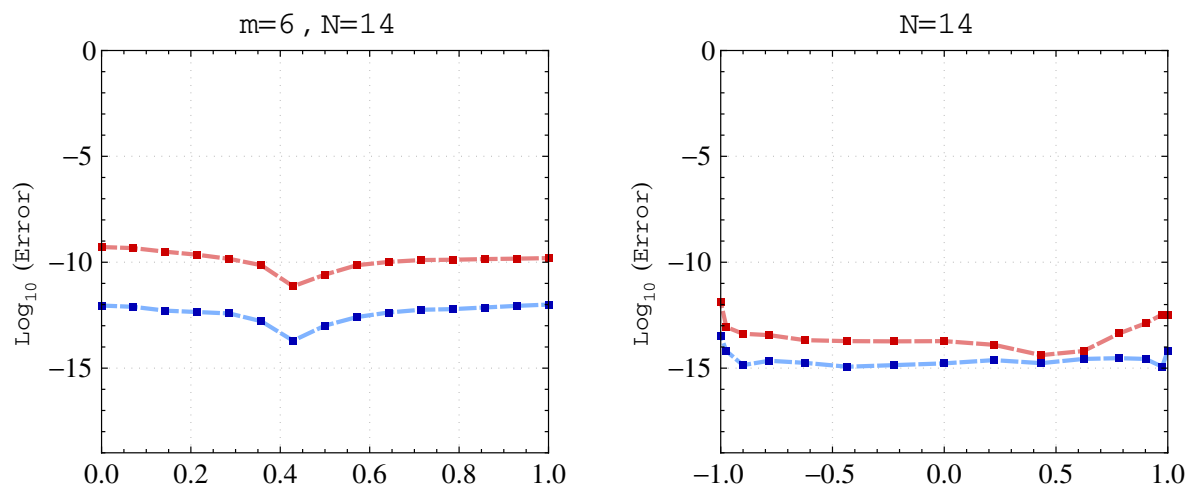

- $\log _{10}|\mathrm{y}-\mathrm{u}|$

- $\log _{10}|z-v|$

Fig. 1. On the left we show the numerical results of the spline collocation method with $N=14$, $c_{m}=1$, and $m=6$ in Example 1. On the right we show the numerical results of the same problem using Jacobi spectral method for $N=14$.
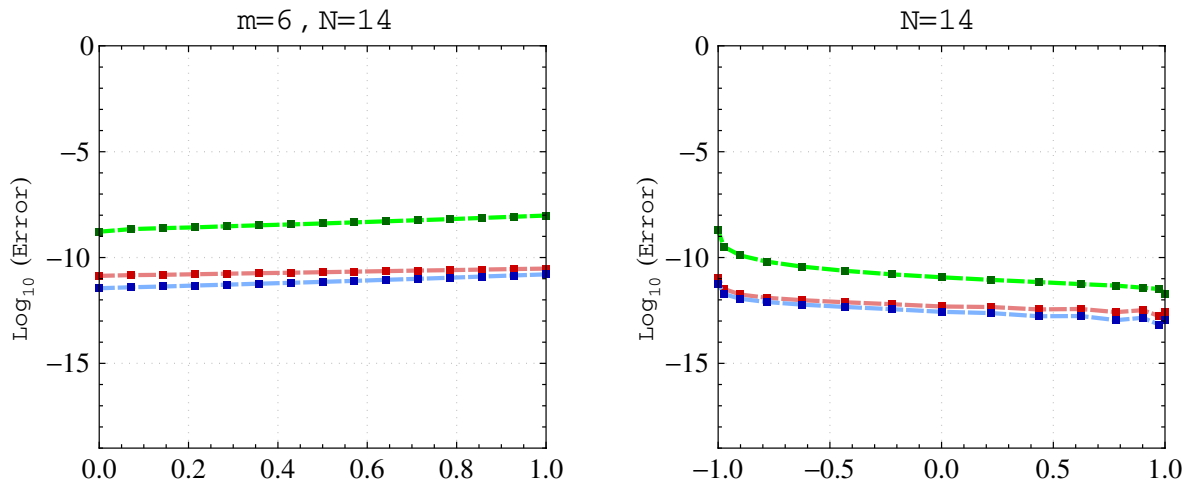

$$
\begin{aligned}
& \log _{10}\left|\mathrm{Y}_{1}-\mathrm{u}_{1}\right| \\
& \log _{10}\left|\mathrm{y}_{2}-\mathrm{u}_{2}\right| \\
& \log _{10}\left|\mathrm{z}_{1}-\mathrm{V}_{1}\right|
\end{aligned}
$$

Fig. 2. On the left we show the numerical results of the spline collocation method with $N=14$, $c_{m}=1$ and $m=6$ in Example 2. On the right we show the numerical results of the same problem using Jacobi spectral method for $N=14$.

where

$$
\begin{gathered}
A=\left(\begin{array}{ll}
1 & 0 \\
0 & 0
\end{array}\right), \quad K(t, s)=\left(\begin{array}{cc}
t+s^{2} & s+t^{2}+1 \\
s^{2}+t^{4}+4 & 0
\end{array}\right), \\
X(t)=(y(t), z(t))^{T}, \quad G(t)=(f(t), g(t))^{T},
\end{gathered}
$$


On the Convergence Analysis of the Spline Collocation Method for System of Integral Algebraic

and $f, g$ such that the exact solution is

$$
y(t)=\frac{t}{t^{4}+1}, \quad z(t)=\arctan \left(t^{2}+1\right) .
$$

The problem is solved by the Jacobi collocation method Hadizadeh et al. [2011], with $\alpha=\frac{1}{2}, \beta=\frac{1}{3}$ and then the $L_{w^{\alpha, \beta}}^{2}$ errors for different values of $N=6,8, \ldots$ are reported in Table 9 . Again, it is solved by the spline collocation method and the maximum errors and order of convergence for different values of $m$ and $N$ at the gridpoints are shown in Tables 10-13. Figure 3 shows a comparison between the spline collocation scheme with $m=6, N=14$ and the Jacobi spectral method for

Table 9. $L_{w^{\alpha, \beta}}^{2}$ errors for Example 3 .

\begin{tabular}{lcc}
\hline$N$ & $\|y-u\|_{L^{\alpha}, \beta}^{2}$ & $\|z-v\|_{L^{\alpha, \beta}}^{2}$ \\
\hline 6 & $6.41 \mathrm{E}-4$ & $7.02 \mathrm{E}-3$ \\
8 & $4.84 \mathrm{E}-5$ & $6.60 \mathrm{E}-4$ \\
10 & $1.97 \mathrm{E}-6$ & $2.59 \mathrm{E}-5$ \\
12 & $2.13 \mathrm{E}-7$ & $1.24 \mathrm{E}-5$ \\
14 & $5.70 \mathrm{E}-8$ & $3.03 \mathrm{E}-6$ \\
\hline
\end{tabular}

Table 10. Maximum errors $\|y-u\|_{\infty}$ and $\|z-v\|_{\infty}$ with $c_{m}=1$ in Example 3 .

\begin{tabular}{lccccccc}
\hline & \multicolumn{3}{c}{$\|y-u\|_{\infty}$} & & \multicolumn{3}{c}{$\|z-v\|_{\infty}$} \\
\cline { 2 - 3 }$m$ & $N=16$ & $N=32$ & $N=64$ & & $N=16$ & $N=32$ & $N=64$ \\
\hline 2 & $1.18 \mathrm{E}-3$ & $2.97 \mathrm{E}-4$ & $7.46 \mathrm{E}-5$ & & $4.90 \mathrm{E}-2$ & $2.53 \mathrm{E}-2$ & $1.28 \mathrm{E}-2$ \\
3 & $2.65 \mathrm{E}-5$ & $3.41 \mathrm{E}-6$ & $4.28 \mathrm{E}-7$ & & $1.55 \mathrm{E}-3$ & $3.78 \mathrm{E}-4$ & $9.45 \mathrm{E}-5$ \\
4 & $8.70 \mathrm{E}-7$ & $5.48 \mathrm{E}-8$ & $3.44 \mathrm{E}-9$ & & $1.18 \mathrm{E}-4$ & $1.52 \mathrm{E}-5$ & $1.94 \mathrm{E}-6$ \\
\hline
\end{tabular}

Table 11. Maximum errors $\|y-u\|_{\infty}$ and $\|z-v\|_{\infty}$ with $c_{m}<1$ in Example 3 .

\begin{tabular}{cccccccc}
\hline & \multicolumn{3}{c}{$\|y-u\|_{\infty}$} & & \multicolumn{3}{c}{$\|z-v\|_{\infty}$} \\
\cline { 2 - 4 } \cline { 7 - 8 }$m$ & $N=16$ & $N=32$ & $N=64$ & & $N=16$ & $N=32$ & $N=64$ \\
\hline 3 & $3.66 \mathrm{E}-5$ & $4.48 \mathrm{E}-6$ & $5.56 \mathrm{E}-7$ & & $1.30 \mathrm{E}-2$ & $6.18 \mathrm{E}-2$ & $3.03 \mathrm{E}-3$ \\
4 & $5.99 \mathrm{E}-6$ & $7.49 \mathrm{E}-7$ & $9.40 \mathrm{E}-8$ & & $1.21 \mathrm{E}-2$ & $6.07 \mathrm{E}-3$ & $3.04 \mathrm{E}-3$ \\
\hline
\end{tabular}

Table 12. Orders of convergence of $u$ and $v$ with $c_{m}=1$ in Example 3.

\begin{tabular}{llllllll}
\hline & \multicolumn{9}{c}{$u$} & & \multicolumn{3}{c}{$v$} \\
\cline { 2 - 3 } \cline { 6 - 7 }$m$ & $N=16$ & $N=32$ & $N=64$ & & $N=16$ & $N=32$ & $N=64$ \\
\hline 2 & 1.97 & 1.99 & 1.99 & & 0.87 & 0.95 & 0.98 \\
3 & 2.90 & 2.95 & 2.99 & & 2.08 & 2.03 & 2.00 \\
4 & 3.91 & 3.98 & 3.99 & & 2.75 & 2.95 & 2.96 \\
\hline
\end{tabular}


Table 13. Orders of convergence of $u$ and $v$ with $c_{m}<1, \rho_{m}=-1$ and $\rho_{m}=1$ in Example 3 .

\begin{tabular}{llllllll}
\hline & \multicolumn{3}{c}{$u$} & & \multicolumn{3}{c}{$v$} \\
\cline { 2 - 3 } \cline { 6 - 8 }$m$ & $N=16$ & $N=32$ & $N=64$ & & $N=16$ & $N=32$ & $N=64$ \\
\hline 3 & 3.08 & 3.03 & 3.01 & & 1.22 & 1.07 & 1.02 \\
4 & 3.01 & 2.99 & 2.99 & & 1.00 & 0.99 & 1.00 \\
\hline
\end{tabular}
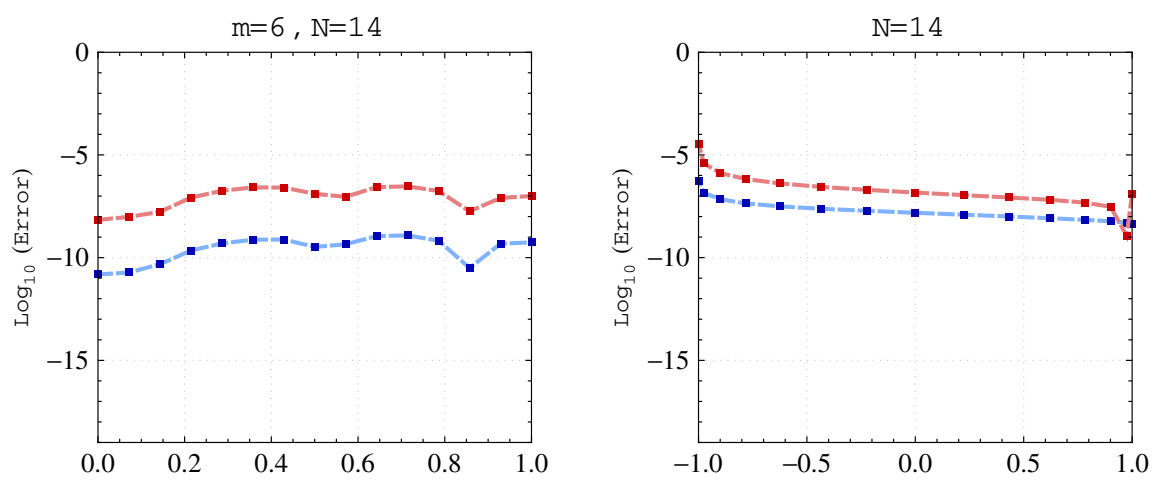

- $\log _{10}|y-u|$

- $\log _{10}|z-v|$

Fig. 3. On the left we show the numerical results of the spline collocation method with $N=14$, $c_{m}=1$ and $m=6$ in Example 3. On the right we show the numerical results of the same problem using Jacobi spectral method for $N=14$.

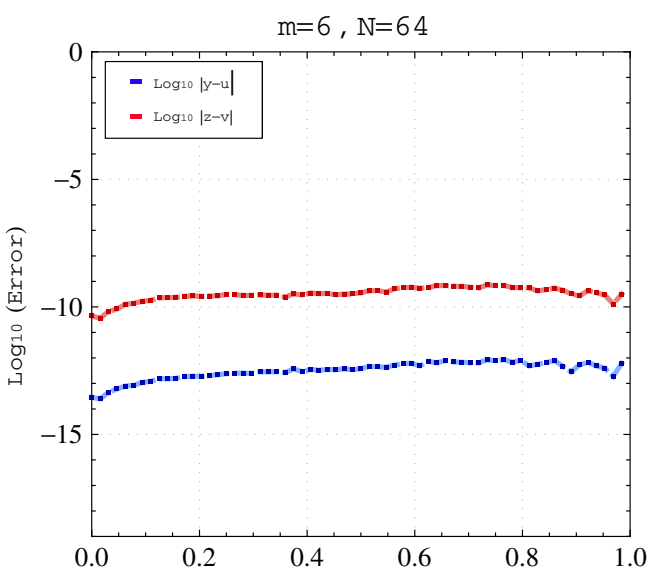

Fig. 4. Numerical results of the spline collocation method with $N=64, c_{m}=1$, and $m=6$ in Example 3. 
$N=14$. As it can be seen from Fig. 3, the spline collocation method is superior in accuracy to the Jacobi spectral method in this case.

Noting that, increasing $m$ in the spline collocation method improve the accuracy, e.g., for $m=6$ and $N=64$, we have

$$
\|y-u\|_{\infty}=8.63 E-13, \quad\|z-v\|_{\infty}=7.26 E-10 .
$$

Figure 4 represents the error behavior for these values of $m$ and $N$.

\section{Conclusion}

In this paper, we performed a polynomial spline collocation method for the numerical solution of system of semi-explicit IAEs of index-2. The most important novelty of this work is obtaining the necessary and sufficient conditions for convergence of discontinuous collocation approximate solution for the IAEs system. We emphasize that in the case of IAEs of index-1, it has shown that the superconvergence occurs when the collocation parameters $c_{j}$ are the Radau $\Pi$ points for $(0,1$ ] [see Kauthen (2000) for further details], however our numerical experiments show that for the system of IAEs of index-2, this property does not occur in these points. More investigation of the superconvergence results for the IAEs of index-2 will be the subject of our future work.

\section{Acknowledgments}

The authors are thankful to Professor Hermann Brunner (Hong Kong Baptist University) for his consideration of the first draft of the manuscript. Also, we would like to acknowledge the referees for their valuable suggestions and comments.

\section{References}

Bernstein, D. S. [2005] Matrix Mathematics: Theory, Facts and Formulas with Application to Linear Systems Theory (Princeton University Press, New Jersey).

Brunner, H. [2004] Collocation Methods for Volterra Integral and Related Functional Equations (Cambridge University Press, New York).

Bulatov, M. V. and Chistyakov, V. F. [1997] "The properties of differential-algebraic systems and their integral analogs," Memorial University of Newfoundland, preprint.

-Bulatov, M. V. and Lima, P. M. [2011] "Two-dimensional integral-algebraic systems: Analysis and computational methods," J. Comput. Appl. Math. 236, 132-140.

Cannon, J. R. [1984] The One-Dimensional Heat Equation (Cambridge University Press, New York).

Chistyakov, V. F. [1986] "On singular systems of ordinary differential equations and their analogues," in Lyapunov Functions and Their Applications (Novosibirsk, NAUKA), pp. 231-240.

Elaydi, S. N. [1999] An Introduction to Difference Equations (Springer-Verlag).

- Gear, C. W. [1988] "Differential-algebraic equations and index transformation," SIAM. J. Stat. Comput. 9, 39-47.

- Gear, C. W. [1990] "Differential-algebraic equations, indices, and integral-algebraic equations," SIAM. J. Numer. Anal. 27, 1527-1534. 
-Gomilko, A. M. [2003] "A Dirichlet problem for the biharmonic equation in a semi-infinite strip," J. Eng. Math. 46, 253-268.

-Hadizadeh, M., Ghoreishi, F. and Pishbin, S. [2011] "Jacobi spectral solution for integralalgebraic equations of index-2," Appl. Numer. Math. 61, 131-148.

-Hairer, E., Lubich, Ch. and Nørset, S. P. [1983] "Order of convergence of one-step methods for Volterra integral equations of the second kind," SIAM. J. Numer. Anal. 20, $569-579$.

- Janno, J. and von Wolfersdorf, L. [1997] "Inverse problems for identification of memory kernels in viscoelasticity," Math. Meth. Appl. Sci. 20, 291-314.

- Jumarhon, B., Lamb, W., McKee, S. and Tang, T. [1996] "A Volterra integral type method for solving a class of nonlinear initial-boundary value problems," Numer. Meth. Partial Diff. Eq. 12, 265-281.

-Kafarov, V. V., Mayorga, B. and Dallos, C. [1999] "Mathematical method for analysis of dynamic processes in chemical reactors," Chem. Eng. Sci. 54, 4669-4678.

Kauthen, J. P. [2000] "The numerical solution of integral-algebraic equations of index-1 by polynomial spline collocation methods," Math. Comput. 236, 1503-1514.

-Wolfersdorf, L. V. [1994] "On identification of memory kernel in linear theory of heat conduction," Math. Meth. Appl. Sci. 17, 919-932.

-Zenchuk, A. I. [2008] "Combination of inverse spectral transform method and method of characteristics: Deformed Pohlmeyer equation," J. Nonlinear Math. Phys. 15, 437-448. 